\title{
JARDINES MEDICINALES EN YUCATÁN: UNA ALTERNATIVA PARA LA CONSERVACIÓN DE LA FLORA MEDICINAL DE LOS MAYAS
}

\author{
MEDICINAL GARDENS IN YUCATÁN: AN ALTERNATIVE FOR THE CONSERVATION \\ OF MEDICINAL FLORA OF THE MAYA
}

\author{
Martha E. Méndez-González ${ }^{\star}$, Wendy M. Torres-Avilez, Alfredo Dorantes-Euán y Rafael Durán-García
}

Centro de Investigación Científica de Yucatán. Calle 43 \# 130.97200, Col. Chuburná. Mérida, Yucatán, México. Tel. 01 (999) 9428330 ext. 216.

${ }^{*}$ Autor para correspondencia (mar@cicy.mx)

\section{RESUMEN}

En Yucatán existe una gran riqueza de conocimiento sobre el uso de las plantas medicinales, que se encuentra en riesgo debido a los actuales procesos de deterioro ambiental y cultural. En este trabajo se da a conocer el establecimiento de jardines comunitarios como una estrategia para salvaguardar el conocimiento que el pueblo maya posee acerca de la flora medicinal y conservar los recursos fitogenéticos de uso medicinal. Se establecieron cuatro jardines medicinales, mediante el método de investigación participativa. Los jardines resguardan el germoplasma de 206 especies, pertenecientes a 170 géneros y 66 familias botánicas; de éstas 160 son especies nativas, de las cuales 11 son endémicas de la Península de Yucatán y 46 son exóticas. Estas especies medicinales se emplean para tratar 301 padecimientos. Las categorías con mayor número de usos son problemas dermatológicos (68), gastrointestinales (59) y reproductivos (33). Estas categorías de uso también incluyeron el mayor número de especies: 82 para problemas dermatológicos, 72 para gastrointestinales, y 35 para los reproductivos y síndromes de filiación cultural. Las especies con mayor valor de uso fueron Aloe vera (L.) Burm. f. (5.5), Ocimum campechianum Mill. (4.75), Tithonia diversifolia (Hemsl.) A. Gray (4.75), Ruta chalepensis L. (4.5), Tradescantia spathacea Sw. (3.5), Parthenium hysterophorus L. (3.25), Chromolaena odorata (L.) R. M. King \& H. Rob. (3.0) y Cissampelos pareira L. (3.0). Las especies con mayor consistencia de uso fueron Tithonia diversifolia para el reumatismo (100\%), y Diphysa carthagenensis Jacq. para el mal de ojo (100\%); además se encontraron 12 especies con una consistencia de uso de $75 \%$. A largo plazo se espera que siguiendo esta estrategia se pueda consolidar una red de jardines medicinales que contribuya a salvaguardar la flora medicinal y el conocimiento asociado al uso de las plantas en esta región del país.

Palabras clave: Conservación de plantas medicinales, usos, médicos tradicionales mayas, jardines medicinales.

\section{SUMMARY}

In Yucatan there is a wealth of knowledge about the use of medicinal plants, which are at risk because of the current processes of cultural and environmental degradation. This work reports on the establishment of community gardens, safeguarding both the knowledge that the Maya people have about medicinal plants and conserving plant genetic resources with medicinal uses. We established four medicinal plant gardens using participatory research methods. These gardens contain 206 species belonging to 170 genera of 66 botanical families; of the 206 species, 46 are exotic, 160 are native species of which 11 are endemic to the Yucatan Peninsula. These medicinal species are employed for treating 301 ailments. The categories with the highest number of uses are skin (68), gastrointestinal (59) and reproductive (33) problems. These categories of uses also included the greatest number of species: 82 for skin problems, 72 for gastrointestinal problems, and 35 species were reported for reproductive problems and for syndromes with a cultural affiliation. The species with the highest use value were Aloe vera (L.) Burm. f. (5.5), Ocimum campechianum Mill. (4.75), Tithonia diversifolia (Hemsl.) A. Gray (4.75), Ruta chalepensis L. (4.5), Tradescantia spathacea Sw. (3.5), Parthenium hysterophorus L. (3.25), Chromolaena odorata (L.) R. M. King \& H. Rob. (3.0) and Cissampelos pareira L. (3.0). Species with greatest use consistency were Tithonia diversifolia used for rheumatism (100\%), and Diphysa carthagenensis Jacq. used for "mal de ojo" (100\%); also we found 12 species with a use consistency of $75 \%$. In the long term we expect to consolidate a network of medicinal plant gardens that contribute to safeguarding the knowledge about the use of medicinal plants, as well as medicinal plants of this region.

Index words: Conservation of medicinal plants, uses, mayan traditional healers, medicinal plant gardens.

\section{INTRODUCCIÓN}

La Península de Yucatán, situada en el sureste de México, se considera una provincia biótica (Morrone, 2005). Aunada a la gran riqueza cultural que aún conserva, esta región cuenta con una flora que aunque no es altamente diversa, está compuesta por elementos de origen centroamericano, caribeño y del sur de México, los que aunados a los elementos endémicos dan como resultado una flora nativa muy peculiar (Durán et al., 1998).

Los mayas peninsulares conservan gran parte de sus costumbres y han conseguido preservar el conocimiento que por siglos han generado acerca del aprovechamiento de su entorno natural, en particular sobre el uso de las plantas medicinales. Este conocimiento se ha transmitido oralmente de generación en generación, aunque en la actualidad existe el riesgo de que estos conocimientos empíricos se pierdan, debido a los cambios sociales y económicos que están viviendo las comunidades mayas y al rápido deterioro de sus ecosistemas naturales (Méndez et al., 2010). 
En el Banco de Datos de Plantas Medicinales de la Península de Yucatán, se tienen registradas para toda la región 680 especies medicinales respaldadas con material herborizado; estas especies están agrupadas en 417 géneros y 119 familias. La entidad de la península que presenta mayor riqueza es Yucatán, con 648 especies distribuidas en 404 géneros y 113 familias, seguido de Quintana Roo con 565 especies distribuidas en 375 géneros y 107 familias botánicas, y Campeche también con 565 especies pero distribuidas en 370 géneros y 107 familias. Las familias de plantas que presentan mayor número de especies medicinales en la región son: Fabaceae, Euphorbiaceae, Asteraceae, Verbenaceae y Solanaceae. Las familias con menor número de especies son: Pinaceae, Rosaceae, Rhizophoraceae, Simaroubaceae y Rhamnaceae (Méndez et al., 2010).

Las plantas medicinales utilizadas por los mayas peninsulares constituyen un recurso no maderable del bosque. Este recurso representa una posibilidad para el uso sustentable de las selvas que aún no ha sido valorado en su total dimensión (Méndez et al., 2010), ya que constituye una alternativa para aliviar los problemas de salud que afectan a la población, por ser fuente potencial para la obtención de nuevos metabolitos que podrían brindar además una opción económica para el desarrollo de proyectos productivos. Es indispensable desarrollar estrategias para el aprovechamiento de este recurso, que permitan preservar el conocimiento que está en riesgo de perderse, conservar el recurso que también está en peligro ante el deterioro de las selvas, así como aprovechar el potencial económico que representa.

Por ello, el desarrollo de colecciones de plantas vivas así como la colecta, caracterización, multiplicación y preservación de propágulos de las especies medicinales, constituyen herramientas importantes para preservar este grupo de plantas. Estas colecciones permiten además avanzar en la documentación de sus usos a fin de conservar el conocimiento asociado a éstas, así como disponer de material genético para su propagación, el restablecimiento de poblaciones de especies amenazadas y en su caso, su aprovechamiento en proyectos productivos.

Los jardines botánicos tienen en México una historia que se remonta a tiempos prehispánicos (Heyden, 2002; Morales-Folguera, 2004). Los antiguos mexicanos establecieron jardines botánicos con una organización definida y un enfoque ecológico, desde el siglo XII, como los jardines fundados por Netzahualcóyotl y Moctezuma (Valdés, 1974), donde las plantas medicinales ocupaban un lugar destacado. En las últimas décadas, el desarrollo de jardines de plantas medicinales se ha fomentado para llevar a cabo el estudio multidisciplinario de la medicina tradicional; tal es el caso del Jardín Etnobotánico del Instituto Nacional de Antropología e Historia (INAH) en Morelos (Avilés, 1985;
Vovides et al., 2010), el Jardín Botánico de Plantas Medicinales Maximino Martínez en la Universidad Autónoma Chapingo (Estrada, 1985) y el Jardín Botánico Medicinal De la Cruz Badiano de la Facultad de Estudios Superiores Zaragoza de la Universidad Nacional Autónoma de México (UNAM), además de otros que cuentan con colecciones de plantas medicinales (Vovides et al., 2010).

El presente trabajo describe el establecimiento de jardines comunitarios, que contribuyen a salvaguardar tanto los recursos fitogenéticos que tienen uso medicinal en la región, como el conocimiento que el pueblo maya posee acerca de su flora medicinal. El establecimiento de los jardines medicinales se enmarcó en la investigación participativa con la comunidad de médicos tradicionales del Estado de Yucatán, a través de la Organización de Médicos Indígenas Mayas de la Península de Yucatán (OMIMPY), lo que permitió incorporar las realidades, necesidades, aspiraciones y creencias de los beneficiarios, al mismo tiempo que los incluyó como protagonistas del proceso (Guzmán-Casado y Alonso-Mielgo, 2007).

\section{MATERIALES Y MÉTODOS}

\section{Área de estudio}

El Estado de Yucatán está situado en el extremo norte de la Península del mismo nombre en el sureste de la República Mexicana. Colinda al norte con el Golfo de México, al este y sureste con Quintana Roo, y al oeste y suroeste con Campeche. El estudio se llevó a cabo en cuatro localidades del Estado de Yucatán: Acanceh (20 48'46” N y $89^{\circ} 27^{\prime} 13^{\prime \prime}$ O), Cantamayec ( $20^{\circ} 28^{\prime} 3^{\prime \prime} \mathrm{N}$ y $\left.88^{\circ} 56^{\prime} 20^{\prime \prime} \mathrm{O}\right)$, Tibolón $\left(20^{\circ}\right.$ $39^{\prime} 55^{\prime \prime} \mathrm{N}$ y $\left.88^{\circ} 56^{\prime} 20^{\prime \prime} \mathrm{O}\right)$ y Yaxcabá $\left(20^{\circ} 32^{\prime} 53^{\prime \prime} \mathrm{N}\right.$ y $88^{\circ}$ $\left.49^{\prime} 38^{\prime \prime} \mathrm{O}\right)$. La región de estudio presenta un clima cálido subhúmedo con lluvias en verano. La temperatura media anual es $26^{\circ} \mathrm{C}$ con máxima de $40{ }^{\circ} \mathrm{C}$ y mínima de $11^{\circ} \mathrm{C}$; la precipitación pluvial media anual oscila entre 700 y 1000 $\mathrm{mm}$. La estación seca es de febrero a mayo, y la lluviosa algunas veces se interrumpe para dar origen a un periodo de sequía conocido como "canícula" la cual ocurre a medio verano. En este territorio no existen corrientes superficiales de agua; sin embargo, el acceso al agua del subsuelo es fácil a través de oquedades naturales comúnmente conocidas como "cenotes". Predominan los suelos someros con fragmentos rocosos clasificados como litosoles y rendzinas (Lugo y García, 1999).

La vegetación predominante en esta zona es la selva baja caducifolia, con un estrato arbóreo que no rebasa los $12 \mathrm{~m}$ de altura y en el cual la familia de las leguminosas es la mejor representada; en el estrato herbáceo abundan gramíneas, compuestas y euforbiáceas; además se pueden encontrar lianas leñosas de la familia Bignoniaceae. Esta comunidad 
se caracteriza por tener un alto porcentaje de individuos que dejan caer sus hojas durante la época seca. Entre las especies más comunes se encuentran Acacia gaumeri S.F. Blake, Alvaradoa amorphoides Liebm., Bauhinia divaricata L., Bursera simaruba (L.) Sarg., Caesalpinia gaumeri Greenm., C. yucatanensis Greenm., Diospyros anisandra S.F. Blake, Guazuma ulmifolia Lam., Havardia albicans (Kunth) Britton \& Rose, Leucaena leucocephala (Lam.) de Wit, Metopium brownei (Jacq.) Urb., Mimosa bahamensis Benth. y Plumeria rubra L. (Olmsted et al., 1999).

De acuerdo con el Instituto Nacional de Estadística y Geografía (INEGI, 2010) Acanceh tiene una población de 10,968 habitantes (5429 hombres, 5539 mujeres); el 28 $\%$ de la población habla la lengua maya. En Cantamayec hay 1695 habitantes (904 hombres, 791 mujeres); en este poblado $80 \%$ de la población es maya hablante. Tibolón cuenta con 1633 habitantes (844 hombres, 789 mujeres), de los cuales $65.4 \%$ hablan la lengua maya, en tanto que la población de Yaxcabá es de 3007 habitantes (1508 hombres, 1499 mujeres), de los cuales casi $56 \%$ son maya hablantes.

\section{Establecimiento de los jardines}

En este estudio se aplicó el método de Investigación Acción Participativa (IAP) (Guzmán-Casado y AlonsoMielgo, 2007). En el año 2011 se llevaron a cabo entrevistas con varios médicos tradicionales para promover con ellos el establecimiento de los jardines. Como resultado de las entrevistas y de acuerdo con el interés mostrado por los médicos tradicionales, se seleccionaron las poblaciones de Acanceh, Cantamayec, Yaxcabá y Tibolón para establecer los jardines medicinales.

El establecimiento de los cuatro jardines se inició en 2011 con la participación de los médicos tradicionales y personas de la comunidad. De acuerdo con las características particulares de cada terreno y de las especies de plantas medicinales que a cada médico tradicional le interesaba conservar, se elaboró un diseño específico para cada jardín, con la participación directa de los médicos tradicionales. En cada jardín se trazaron y construyeron caminos para facilitar el acceso a las plantas, así como su exhibición, como se muestra en la Figura 1. Cuando fue necesario también se perforaron pozos para asegurar el abastecimiento de agua.

Se llevó a cabo un registro de las especies medicinales que ya estaban establecidas en el terreno. Se realizó la colecta, identificación e incorporación de los ejemplares de estas especies al herbario del Centro de Investigación Científica de Yucatán. Además, se aplicaron entrevistas semiestructuradas a los médicos tradicionales responsables de los jardines (uno por cada jardín), con lo cual se obtuvo la información de sus prácticas curativas y datos etnobotánicos asociados a cada especie; con cuya información se elaboró una base de datos.

Una vez establecida la infraestructura básica de cada jardín, se llevó a cabo la colecta de propágulos, su propagación e introducción, de las plantas de especies medicinales de interés para cada médico tradicional responsable del jardín. De forma complementaria, en cada jardín se organizó un taller de intercambio entre los médicos tradicionales, tanto de información como de germoplasma, para enriquecer las colecciones de los jardines medicinales y que todos los participantes conocieran los otros jardines que se estaban estableciendo.

\section{Base de datos}

Con la información obtenida se construyó una base de datos mediante el programa Microsoft Excel 2010@. La base consta de tres apartados: taxonómico (familia, género, especie y registro de los ejemplares, como autor, herbario, colector y número de colecta); geográfico (localidad de la encuesta y distribución de la especie); y etnobotánico (nombre común, padecimiento, categoría del padecimiento, estructura vegetal empleada, receta y dosis).

Para determinar la distribución de las especies se consultó la Flora de Guatemala, Flora de La Española, Flora Mesoamericana y Flora de Nicaragua. También se revisó la base de datos de TROPICOS y la de GRIN (Germoplasm Resources Information Network) del Departamento de Agricultura de los Estados Unidos de América. La distribución de las especies se clasificó en endémica, nativa y exótica; las especies endémicas son aquellas cuya distribución se restringe a la Península de Yucatán; nativas, las especies que se encuentran de forma natural en la Península de Yucatán pero tienen una distribución más amplia; y exóticas aquéllas cuya distribución natural no abarca el territorio de la Península.

Los padecimientos que se reportaron se clasificaron en 11 categorías en función del aparato (i.e. digestivo, circulatorio) o sistema (i.e. nervioso) al que afectan. Se agregaron tres categorías que no están relacionadas directamente con un aparato o sistema; tal es el caso de la categoría de otros (que incluye aquellos que no se ubican en las categorías anteriores, como mordedura de víbora), signos y síntomas (los que no corresponden a un padecimiento único sino que pueden corresponder a diversos padecimientos, como el dolor de cabeza) y sindrome de filiación cultural que incluye a los padecimientos que se encuentran bajo el contexto cultural propio de cada grupo, para los cuales es evidente el uso de procedimientos de eficacia simbólica para recuperar la salud (Zolla et al., 1988; Aguilar-Contreras et al., 1998; Fagetti, 2004). 


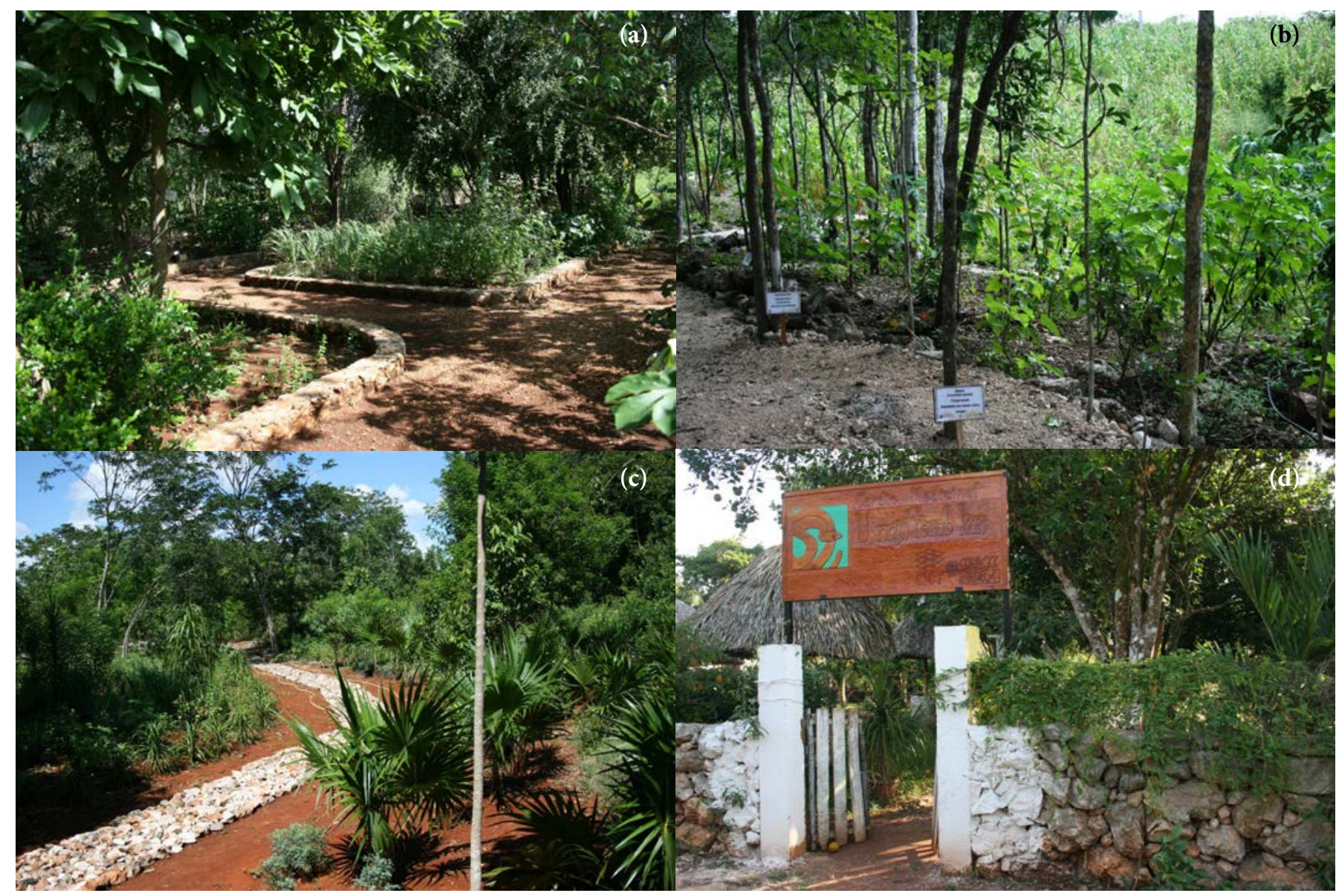

Figura 1. Jardines de plantas medicinales establecidos en Acanceh (a), Cantamayec (b), Yaxcabá (c) y Tibolón (d), en el Estado de Yucatán, México.

\section{Análisis de la información}

Se determinó el número de familias, géneros y especies con uso medicinal en cada jardín, la forma de crecimiento de las especies, su distribución y se contabilizó el número de especies para cada categoría de padecimiento.

Se calculó el valor de uso $(V U)$ de las especies en los cuatro jardines, con la fórmula $V U=\Sigma U / n$ propuesta por Phillips et al. (1994) y modificada por Rossato et al. (1999), donde $\Sigma U$ es la sumatoria del número de usos de una especie mencionada en los jardines y $n$ es el total de jardines. También se determinó la consistencia de uso $(C U)$ de las especies que muestra cuales son las especies que se emplean para el mismo padecimiento en los cuatro jardines, mediante la fórmula: $C U=\sum i u / N \times 100$, donde $\sum i u$ es la sumatoria de informantes que usan una especie para un padecimiento en específico y $\mathrm{N}$ es el total de informantes.

Además se calculó el índice de similitud con el total de recursos vegetales empleados entre los jardines para ello se utilizó el índice de Sørensen (Mueller-Dombois y Ellenberg, 1974): $I S s=(2 c / A+B) \times 100$, donde $c$ es la suma del número de especies comunes para ambos grupos; $A$ es el número de especies para el grupo uno, y $B$ es el número de especies para el grupo dos.

\section{RESULTADOS Y DISCUSIÓN}

En 2012 se concluyó el establecimiento de cuatro jardines medicinales, cada uno a cargo de un médico tradicional de la localidad, que resguardan el germoplasma de 206 especies con uso medicinal, pertenecientes a 170 géneros, de $66 \mathrm{fa}$ milias botánicas empleadas por las comunidades donde los jardines se ubican. Del total de especies, 160 son especies nativas de las cuales 11 son endémicas de la Península de Yucatán y 46 corresponden a especies exóticas (ver lista completa de especies en: "http://www.revistafitotecniamexicana.org/ documentos/37-2/anexo1.pdf"). El total de especies que resguardan los jardines representa casi la tercera parte de las 648 especies con uso medicinal registradas para el Estado de Yucatán (Méndez et al., 2010). En estos jardines medicinales 
se conserva el germoplasma tanto in situ como ex situ, ya que algunas especies están en su hábitat natural, en tanto que otras aunque nativas de Yucatán, fueron sembradas en el jardín al igual que las exóticas, que no crecen de manera natural en la región, pero los médicos mayas las consideran especies importantes.

Las diez familias con mayor número de especies son Fabaceae (23), Euphorbiaceae (13), Asteraceae (11), Malvaceae (11), Lamiaceae (9), Apocynaceae (7), Boraginaceae (7), Bignoniaceae (6), Rutaceae (6) y Solanaceae (6). Al respecto, las cuatro familias con mayor número de especies coinciden con las reportadas en un estudio que incluyó todo el Estado de Yucatán (Méndez et al., 2010), así como con estudios realizados en otras partes del mundo como China, Etiopía, Perú, entre otros (De-la-Cruz et al., 2007; Teklehaymanot, 2009; Weckerle et al., 2009), lo cual resulta comprensible, considerando que estas familias son las más diversas en el mundo (Heywood, 1993).

El jardín de plantas medicinales de Acanceh "X ka' ansajil" (Lugar de enseñanza) está bajo la responsabilidad de Don Edgar Francisco Peraza Chan; este jardín forma parte de la Brigada de Desarrollo Rural No. 73 y en media hectárea resguarda el germoplasma de 96 especies con uso medicinal, pertenecientes a 87 géneros y 44 familias botánicas. Del total de especies, 69 son nativas, cuatro de las cuales son endémicas, y 27 son especies exóticas. Las familias con mayor número de especies son Euphorbiaceae (9), Asteracaeae y Fabaceae (7).

El jardín de Cantamayec “Ts’u kaax u bu'tuni ti tsak” (El cerro de las medicinas), está a cargo de Don Asunción Cetina Chan. Este jardín cuenta con una hectárea donde se tienen 123 especies pertenecientes a 111 géneros de 52 familias botánicas. Del total de especies, 107 son nativas, siete de ellas son endémicas y 16 son especies exóticas. Las familias botánicas con mayor número de especies son Fabaceae (19), Euphorbiaceae (9), Apocynaceae, Boraginaceae, Lamiaceae y Malvaceae (5).

En Tibolón "U Najil Tsaab Kan" (La casa de la serpiente cascabel), a cargo de Don Carmito Antonio Ek Catzin, el jardín resguarda en media hectárea el germoplasma de 53 especies pertenecientes a 53 géneros y 33 familias botánicas. El número de especies nativas es 41 , de éstas una es endémica, y además cuenta con 12 especies exóticas. Las familias Asteraceae (6), Euphorbiaceae y Fabaceae (4) son las que presentan el más alto número de especies.

El jardín de Yaxcabá "U kuchil xiw kuts' aak" (El lugar de las plantas medicinales), a cargo de Don Juan Bautista Cob Balam, resguarda en casi dos hectáreas el germoplasma de 109 especies pertenecientes a 97 géneros y 47 familias. De este total, 75 son nativas, siete de las cuales son endémicas, más 34 exóticas. Las familias con mayor número de especies son Fabaceae (11), Asteraceae (9), Lamiaceae y Malvaceae (6).

La mayor parte $(78 \%)$ de las especies que los jardines medicinales resguardan corresponden a especies nativas, lo cual refleja el amplio conocimiento que los médicos tradicionales responsables de los jardines poseen sobre el uso de los recursos vegetales de su entorno. Del total de especies medicinales que los cuatro jardines agrupan, $30 \%$ corresponde a plantas herbáceas, $29 \%$ son árboles y $25 \%$ arbustos; el $16 \%$ restante corresponde a otras formas de vida. En este estudio, al igual que en otros (Voeks, 1996), las hierbas son elementos importantes dentro de los recursos vegetales medicinales empleados por diferentes grupos, pues no requieren de mucho espacio para establecerlas, tienen un ciclo de vida corto y generalmente están rápidamente disponibles. Además, se han reportado muchas herbáceas con altos contenidos de componentes bioactivos (Stepp y Moerman, 2001; Stepp, 2004).

Las especies reportadas se emplean para tratar 301 padecimientos. Las categorías para las cuales se reporta un alto número de usos son para problemas dermatológicos (68), gastrointestinales (59) y reproductivos (33). Estas categorías de uso también incluyeron el mayor número de especies; 82 para problemas dermatológicos, 72 para gastrointestinales; para problemas reproductivos y para tratar los síndromes de filiación cultural se emplean 35 especies.

En el Cuadro 1 se resalta la diversidad de especies que se emplean para atender los problemas gastrointestinales y dermatológicos en los cuatro jardines. Las especies que se emplean en un mayor número de padecimientos, son Aloe vera (L.) Burm. f., Ocimum basilicum L., Cissampelos pareira L., Tithonia diversifolia (Hemsl.) A. Gray, Ipomoea carnea Jacq., Ocimum campechianum Mill., Ruta chalepensis L., Azadirachta indica A. Juss y Tradescantia spathacea Sw. (Cuadro 2).

Ankli et al. (1999) y Méndez et al. (2010) también reportan para el Estado de Yucatán que el mayor número de especies con uso medicinal son empleadas para atender los problemas dermatológicos y gastrointestinales. Cabe mencionar que en México, las enfermedades infecciosas y parasitarias se encuentran entre las primeras seis causas de muerte en niños menores de 5 años (Secretaría de SaludYucatán, 2007; Sistema Nacional de Vigilancia Epidemiológica, 2010); la incidencia es aún mayor en las zonas rurales y en las comunidades indígenas (Méndez-González y Cervera-Montejano, 2006).

Los problemas dermatológicos, aunque no presentan tasas 
Cuadro 1. Categorías de uso con mayor número de especies vegetales en cada jardín de plantas medicinales.

\begin{tabular}{lcccc}
\hline Categoría de uso & Acanceh & Cantamayec & Tibolón & Yaxcabá \\
\hline Gastrointestinales & 23 & 49 & 20 & 20 \\
Dermatológicos & 29 & 37 & 17 & 27 \\
Metabólicos & 17 & & 9 & \\
Músculo esqueléticos & & & 9 & 16 \\
Reproductivos & & & \\
Respiratorios & & & 20 \\
Signos y síntomas & & & \\
Síndromes de filiación cultural & & & & \\
\hline
\end{tabular}

Cuadro 2. Especies que se utilizan en un mayor número de padecimientos en cada jardín de plantas medicinales.

\begin{tabular}{|c|c|c|c|c|}
\hline Especies & Acanceh & Cantamayec & Tibolón & Yaxcabá \\
\hline Aloe vera (L.) Burm. f. & 9 & & 5 & \\
\hline Cissampelos pareira $\mathrm{L}$. & & 9 & & \\
\hline Ocimum basilicum $\mathrm{L}$. & 9 & & & \\
\hline Tithonia diversifolia (Hemsl.) A. Gray & & & 9 & \\
\hline Ipomoea carnea Jacq. & & 8 & & \\
\hline Ocimum campechianum Mill. & & 8 & 5 & \\
\hline Ruta chalepensis L. & 7 & & & 8 \\
\hline Azadirachta indica A. Juss & 7 & & & \\
\hline Tradescantia spathacea Sw. & 7 & & & \\
\hline Chromolaena odorata (L.) R. M. King \& H. Rob. & & 6 & & \\
\hline Elytraria imbricata (Vahl) Pers. & & 6 & & \\
\hline Jatropha gaumeri Greenm. & & 6 & & \\
\hline Parthenium hysterophorus L. & & & 6 & 4 \\
\hline Persea americana Mill. & & & 5 & \\
\hline Bunchosia swartziana Griseb. & & & & 4 \\
\hline Mentha citrata Ehrh. & & & & 4 \\
\hline Urera baccifera (L.) Gaudich. ex Wedd. & & & & 4 \\
\hline
\end{tabular}

altas de mortalidad, son muy comunes y si no se atienden adecuadamente pueden conducir a problemas de salud más severos, además de que constituyen una de las principales causas de atención primaria de las zonas tropicales (Roderick et al., 2006). El alto número de especies para tratar problemas gastrointestinales refleja la búsqueda de recursos vegetales alternativos a la medicina alopática, para solventar estos problemas de salud en las comunidades mayas de Yucatán, sobre todo en los niños que se ven afectados recurrentemente por este tipo de padecimientos.

Si bien todas las especies de la flora medicinal constitu- yen un patrimonio biocultural del pueblo maya, en particular las especies empleadas para tratar los síndromes de filiación cultural, también son interesantes porque su uso está acompañado de rituales que conllevan creencias mágico-religiosas y refuerzan los elementos culturales, cosmovisiones y saberes ancestrales del pueblo maya en constante defensa ante los embates de la cultura hegemónica. En este estudio, 35 especies son empleadas para tratar síndromes de filiación cultural como cirro, mal de ojo, mal viento, empacho, pasmo, etc.

Con base en los usos reportados por los informantes 
de cada jardín, en el Cuadro 3 se presentan las ocho especies con mayor valor de uso. Para varias especies se han reportado actividades biológicas diversas, algunas de las cuales están relacionadas con el uso tradicional reportado. Tal es el caso de Tradescantia spathacea para la que se ha reportado acción sedante (Germosén-Robineau, 2005), estimulante de útero (Weniger et al., 1982), antiinflamatoria (Pérez, 1996), actividad citotóxica contra células cancerígenas (Mena-Rejón et al., 2009), antioxidante (González-Avila et al., 2003) y protección contra cáncer de hígado (Rosales-Reyes et al., 2008); los médicos tradicionales reportan su uso para la fertilidad, duchas vaginales, infección vaginal y el pasmo, además de aplicarla contra el cáncer.

En los jardines se encuentran 46 especies exóticas que se utilizan en la medicina maya. Pieroni et al. (2005) y Ceuterick et al. (2008) mencionan que las migraciones de los grupos humanos tienen influencia en la conformación de las farmacopeas locales al incorporar remedios medicinales provenientes de diferentes partes del mundo. En el caso de la flora medicinal maya, algunas de estas especies exóticas, no sólo se incorporaron a la farmacopea local, sino que además tienen un alto valor de uso, como ocurre con Aloe vera y Ruta chalepensis.

La consistencia de uso y el valor de uso pueden indicar la eficacia de ciertas especies para satisfacer los problemas de salud de un grupo humano. Este indicador podría ser una guía para seleccionar especies que pueden someterse a pruebas de actividad biológica, lo que eventualmente podría

Cuadro 3. Especies de los jardines medicinales con mayor valor de uso y padecimientos en los que se emplean.

\begin{tabular}{lll}
\hline Especie & Valor de uso & \multicolumn{1}{c}{ Padecimientos } \\
\hline Aloe vera & $\begin{array}{l}\text { Caída da cabello, calvicie, cáncer, caspa, colesterol, diabetes, dolor de } \\
\text { espalda, dolor de muela, entumecimiento del cuerpo, gastritis, hemo- } \\
\text { roides, hinchazón del cuerpo, llagas, reumatismo, como cicatrizante } \\
\text { y desinflamatorio. }\end{array}$
\end{tabular}

Ocimum campechianum

Tithonia diversifolia

4.75

Ruta chalepensis

Tradescantia spathacea

Parthenium hysterophorus

Chromolaena odorata

3.0

Cissampelos pareira

3.0
Cáncer en el estómago, cáncer en la sangre, cicatrizante de granos y heridas, disentería, fuego de San Lázaro, gastritis, heridas en diabéticos, tumores, hongos y viruela.

Asma, bronquitis, cuerpo frío en niños, dejar de fumar, diabetes, dolor de estómago, dolor de pies, dolor muscular, fuego de San Lázaro, granos, hemorroides, incordio, reumatismo, tos y viruela.

Alteración de nervios, cólico, después del parto, dolor de cabeza, dolor muscular, hongos en el cuerpo, limpias, mal aire, mal de ojo, niños que sudan mucho en la noche, reumatismo, salpullido, santiguar, susto y vómito.

Ardor en el cuerpo, asma, bronquitis, cáncer, dolor de cabeza, duchas vaginales, fertilidad, flujo vaginal, gastritis, golpes, manchas en la piel, pasmo y tos.

Alteración de nervios, cicatrizante de heridas, dolor muscular, dolores reumáticos, fuego de San Lázaro, granos, hemorroides, hongos, mal aire, menopausia, reumatismo y viruela.

Dolor al orinar, gonorrea, infección vaginal, infección en los riñones, llagas en el cuerpo, piedras en los riñones, próstata, salpullido y tensión en la vejiga.

Cólicos, diabetes, diarrea, diarrea blanca, disentería, dolor de estómago con cólico y agruras, estreñimiento, gastritis, heridas de diabéticos y mal de ojo. 
conducir al descubrimiento de nuevos fármacos. También se podría corroborar científicamente el uso de esas especies a fin de que las personas lo puedan utilizar con criterios de eficacia y seguridad, como lo ha hecho el proyecto TRAMIL (Programa para la Investigación Aplicada y la Difusión de Plantas Medicinales en el Caribe) por casi tres décadas (Germosén-Robineau, 2005).

El análisis de la consistencia de uso muestra dos especies con $100 \%$ de consistencia: Tithonia diversifolia para el reumatismo y Diphysa carthagenensis Jacq. para el mal de ojo. Además, se reportan otras 12 especies con una consistencia de uso de 75 \%: Abrus precatorius L. (mal de ojo), Aloe vera (diabetes), Asclepias curassavica L. (granos), Cecropia peltata L. (diabetes), Cnidoscolus aconitifolius (Mill.) I.M. Johnst. (piedras en los riñones), Mimosa pudica L. (insomnio), Pedilanthus itzaeus Millsp. (reumatismo), Plectranthus amboinicus (Lour.) Spreng. (asma), Senna occidentalis (L.) Link (asma), Tecoma stans (L.) Juss. ex Kunth (diabetes), Teucrium cubense Jacq. (diarrea) y Thevetia ahouai (L.) A. DC. (reumatismo).

Se observó una similitud de $61 \%$ entre los jardines de Acanceh y Yaxcabá, los cuales comparten 63 especies, mientras los más disimiles son los jardines de Tibolón y Acanceh con $28 \%$ de similitud, ya que sólo comparten 31 especies. Por otra parte existen especies exclusivas de cada jardín: en Acanceh hay 19 especies exclusivas, el de Cantamayec tiene nueve, en Tibolón seis, y el de Yaxcabá cuenta con 25 (Cuadro 4). Los análisis de similitud de los jardines medicinales, en relación con las especies que se establecen en cada jardín, son necesarios para lograr un buen manejo de ellos, ya que permite conocer cuáles son las especies exclusivas de cada jardín y cuáles son las que se comparten entre ellos, lo que impulsa el intercambio de recursos vegetales entre los jardines.

El establecimiento y consolidación de estos jardines medicinales pretende ser la base para una eventual Red de Jardines Medicinales del Mayab, que apoye la documentación de los usos de las plantas medicinales de esta región y contribuya a la conservación de la flora medicinal maya y el conocimiento asociado a ella. De esta manera, es posible afrontar mejor los riesgos que representa la transmisión del conocimiento solo a través de la tradición oral, así como salvaguardar la flora medicinal ante la crisis ambiental que pone en riesgo la diversidad biológica y erosiona la calidad de los ecosistemas y los servicios ambientales que proporcionan.

\section{CONCLUSIONES}

Mediante el método de investigación participativa para el establecimiento de cuatro jardines medicinales, se está promoviendo la conservación de casi una tercera parte de las especies que conforman el recurso vegetal con uso medicinal en el Estado de Yucatán, ya que éstos cuentan con al menos 206 especies de las 648 que a la fecha se tienen registradas en la entidad. Con la documentación del conocimiento asociado al uso de estas especies, aportada por los médicos tradicionales indígenas, se contribuye a la conservación de este valioso saber. El que los médicos tradicionales estén al frente de los jardines contribuye a garantizar su permanencia, ya que ellos son los promotores terapéuticos y principales usuarios de este recurso; esto es acorde con la idea de que la conservación in situ de los recursos naturales sólo podrá lograrse en la medida de que los usuarios de estos recursos sean sus principales defensores y esto repercuta en el reforzamiento de su cultura e identidad.

Las categorías de padecimientos para los que se registraron mayor número de especies fueron los problemas gastrointestinales y dermatológicos. Las especies nativas son las más utilizadas por los médicos mayas y constituyen 78 $\%$ de las especies que usan y resguardan estos jardines medicinales.

Las especies con mayor valor de usos fueron Aloe vera, Ocimum campechianum, Tithonia diversifolia, Ruta chalepensis, Tradescantia spathacea, Parthenium hysterophorus, Chromolaena odorata y Cissampelos pareira. Los análisis de valor de uso y consistencia de uso proporcionaron información sobre las especies medicinales más importantes, tanto para conservar como para estudiar desde una perspectiva química, farmacológica, toxicológica, etc. El índice de similitud proporciona información para hacer el intercambio tanto de conocimiento como de los recursos vegetales.

El establecimiento de jardines medicinales constituye parte de una estrategia para la conservación de los recursos fitogenéticos de uso medicinal empleados hoy en día por los mayas de esta región, tanto in situ como ex situ.

\section{AGRADECIMIENTOS}

A Don Edgar Francisco Peraza Chan, Don Asunción Cetina Chan, Don Carmito Antonio Ek Catzin y Don Juan Bautista Cob Balam, por compartir su conocimiento sobre las plantas medicinales, ya que sin ellos este estudio no hubiese sido posible. A FORDECYT-CONACYT por su financiamiento a través del proyecto No. 115911. A José Luis Tapia, Paulino Simá y Filogonio May, por su ayuda en la determinación de los ejemplares botánicos, así como a Topacio Pacheco, María Pía Mc Manus, Gerardo Godoy, Hiram Blancarte y Fernando Fleites, por su contribución en el trabajo de campo. 
Cuadro 4. Especies exclusivas de cada jardín medicinal.

\begin{tabular}{|c|c|c|c|}
\hline Tibolón & Cantamayec & Acanceh & Yaxcabá \\
\hline Costus pictus & Abrus precatorius & Agave fourcroydes & Abelmoschus esculentus \\
\hline Euphorbia schlechtendalii & Alvaradoa amorphoides & Agave sisalana & Allium cepa \\
\hline Manilkara zapota & Elytraria imbricata & Anacardium occidentale & Allium sativum \\
\hline Rivina humilis & Dorstenia contrajerva & Argemone mexicana & Bougainvillea glabra \\
\hline Sambucus canadensis & Hamelia patens & Bromelia pinguin & Bromelia karatas \\
\hline \multirow[t]{20}{*}{ Zingiber officinale } & Lepidium virginicum & Ceiba ae sculifolia & Bursera graveolens \\
\hline & Lippia graveolens & Coccoloba uvifera & Caesalpinia yucatanensis \\
\hline & Senna occidentalis & Crescentia alata & Cedrela odorata \\
\hline & Spondias mombin & Cyrtopodium macrobulbon & Chrysophyllum cainito \\
\hline & & Kalanchoe delagoensis & Cochlospermum vitifolium \\
\hline & & Lonchocarpus punctatus & Cohniella ascendens \\
\hline & & Nicotiana tabacum & Cordia sebestena \\
\hline & & Pouteria campechiana & Croton chichenensis \\
\hline & & Rosmarinus officinalis & Datura inoxia \\
\hline & & Selaginella convoluta & Ehretia tinifolia \\
\hline & & Spondias purpurea & Hibiscus poeppigii \\
\hline & & Thrinax radiata & Indigofera suffruticosa \\
\hline & & Walteria indica & Lycopersicon esculentum \\
\hline & & Zea mays & Mentha citrata \\
\hline & & & Montanoa grandiflora \\
\hline & & & Phyla stoechadifolia \\
\hline & & & Phyllanthus glaucescens \\
\hline & & & Terminalia catappa \\
\hline & & & Zanthoxylum fagara \\
\hline & & & Zinnia violacea \\
\hline
\end{tabular}

\section{BIBLIOGRAFÍA}

Aguilar-Contreras A., J. R. Camacho-Pulido, S. Chino-Vargas, P. Jaquez-Ríos y M. E. López-Villafranco (1998) Plantas Medicinales del Herbario IMSS: Su Distribución por Enfermedades. Ed. Instituto Mexicano del Seguro Social. México, D. F. 167 p.

Ankli A., O. Sticher and M. Heinrich (1999) Medical ethnobotany of the Yucatec Maya: healers consensus as a quantitative criterion. Economic Botany 53:144-160.

Avilés M. (1985) Jardines botánicos en la cultura: Jardín etnobotánico del INAH-Centro regional Morelos. In: Memorias de la Primera Reunión Nacional de Jardines Botánicos. Ed. Instituto Nacional de Ecología. México, D. F. pp:27-31.

Ceuterick M., I. Vandebroek, B. Torry and A. Pieroni (2008) Crosscultural adaptation in urban ethnobotany: the Colombian folk pharmacopoeia in London. Journal of Ethnopharmacology 120:342-359.

De-la-Cruz H., G. Vilcapoma and P. A. Zevallos (2007) Ethnobotanical study of medicinal plants used by the Andean people of Canta, Lima, Peru. Journal of Ethnopharmacology 111:284-294.

Durán R., J. C. Trejo and G. Ibarra-Manríquez (1998) Endemic phytotaxa of the peninsula of Yucatan. Harvard Papers in Botany 3:263-314.

Estrada E. (1985) Jardín botánico de plantas medicinales Maximino Martínez. In: Memorias de la Primera Reunión Nacional de Jardi- nes Botánicos. Ed. Instituto Nacional de Ecología. México, D F. pp:69-83.

Fagetti A. (2004) Síndromes de Filiación Cultural: Conocimiento y Práctica de los Médicos Tradicionales en Cinco Hospitales Integrales con Medicina Tradicional del Estado de Puebla. Secretaría de Salud, Gobierno del Estado de Puebla. Puebla, México. 170 p.

Germosén-Robineau L. (2005) Farmacopea Vegetal Caribeña. Ed. Universitaria. Universidad Nacional Autónoma de Nicaragua. León, Nicaragua. 486 p.

González-Ávila M., M. Arriaga-Alba, M. De-la-Garza, M. del C. Hernández-Pretelín, M. A. Domínguez-Ortiz, S. Fattel-Fazenda y S. Villa-Treviño (2003) Antigenotoxic, antimutagenic and ROS scavenging activities of a Rhoeo discolor ethanolic crude extract. Toxicology in Vitro 17:77-83.

Guzmán-Casado G. I. y A. M. Alonso-Mielgo (2007) La investigación participativa en agroecología: una herramienta para el desarrollo sustentable. Ecosistemas 16:24-36.

Heyden D. (2002) Jardines botánicos prehispánicos. Arqueología Mexicana 10:18-23.

Heywood V. H. (1993) Flowering Plants of the World. Oxford Univ. Press. New York, USA. 335 p.

INEGI, Instituto Nacional de Estadística, Geografía e Informática (2010) Censo de población y vivienda 2010. Disponible en :http://www3.inegi.org.mx/sistemas/mexicocifras/ (noviembre 2013). 
Lugo J. y M. T. García (1999) Geomorfología de la Península de Yucatán. In: Atlas de los Procesos Territoriales de Yucatán. P. ChicoPonce-de-León y A. García (eds). Universidad Autónoma de Yucatán, Mérida, Yucatán, México. pp:155-162.

Mena-Rejón G., E. Caamal-Fuentes, Z. Cantillo-Ciau, R. Cedillo-Rivera, J. Flores-Guido and R. Moo-Puc (2009) In vitro cytotoxic activity of nine plants used in Mayan traditional medicine. Journal of Ethnopharmacology 121:462-465.

Méndez M., R. Durán, S. Campos y A. Dorantes (2010) Flora medicinal: In: Biodiversidad y Desarrollo Humano en Yucatán. R. Durán y M. Méndez (eds). Ed. Centro de Investigación Científica de Yucatán. Programa de Pequeñas Donaciones del Fondo para el Medio Ambiente Mundial. Comisión Nacional para el Conocimiento y Uso de la Biodiversidad. Secretaría de Desarrollo Urbano y Medio Ambiente de Yucatán. Mérida, Yucatán, México. pp:349-352.

Méndez-González R. M. y M. D. Cervera-Montejano (2006) Análisis espacial de mortalidad y servicios de salud en municipios indígenas de Yucatán, México, 1990-2000. Revista Biomédica 17:108-117.

Morales-Folguera J. M. (2004) Jardines prehispánicos de México en las crónicas de Indias. Archivo Español de Arte 77:351-373.

Morrone J. J. (2005) Hacia una síntesis biogeográfica de México. Revista Mexicana de Biodiversidad 72:207-252.

Mueller-Dombois D. and H. Ellenberg (1974) Aims and Methods of Vegetation Ecology. Wiley Int. New York, USA. 547 p.

Olmsted I., J. A. González-Iturbe, J. Granados-Castellanos, R. Durán y F. Tun-Dzul (1999) Vegetación de la Península de Yucatán. In: A. García de Fuentes, J. Córdoba, P. Chico (eds.). Atlas de Procesos Territoriales de Yucatán. Mérida, Yucatán, México. pp:183-194.

Pérez R. M. (1996) Anti-inflammatory activity of Ambrosia artemisiaefolia and Rhoeo spathacea. Phytomedicine 3:163-167.

Phillips O. L., A. H. Gentry, C. Reynel, P. Wilkin and C. Galvez-Durand (1994) Quantitative ethnobotany and Amazonian conservation. Conservation Biology 8:225-248.

Pieroni A., H. Muenz, M. Akbulut, K. Baser and C. Durmuşkahya (2005) Traditional phytotherapy and trans-cultural pharmacy among Turkish migrants living in Cologne, Germany. Journal of Ethnopharmacology 102:69-88.

Roderick H., S. E. Bendeck, S. Chen, R. Estrada, A. Haddix, T. McLeod and A. Mahé (2006) Skin diseases. In: Disease Control Priorities in Developing Countries. T. D. Jamison, J. G. Breman, A.
R. Measham, G. Alleyne, M. Claeson, D. B. Evans, P. Jha, A. Mills, P. Musgrove (eds). World Bank. Washington, DC, USA. pp:707-721.

Rosales-Reyes T., M. De-la-Garza, C. Arias-Castro, M. RodríguezMendiola, S. Fattel-Fazenda, E. Arce-Popoca, S. HernándezGarcía and S. Villa-Treviño (2008) Aqueous crude extract of Rhoeo discolor, a Mexican medicinal plant, decreases the formation of liver preneoplastic foci in rats. Journal of Ethnopharmacology 115:381-386.

Rossato S. C., H. F. Leitão-Filho and A. Begossi (1999) Ethnobotany of Caiçaras of the Atlantic forest coast (Brazil). Economic Botany 53:387-395.

Secretaría de Salud-Yucatán (2007) Sistema Epidemiológico y Estadístico de Defunciones. Disponible en: http://www.salud.yucatan. gob.mx/images/stories/pdf/ INFANTIL2007.pdf. (Noviembre 2013).

Sistema Nacional de Vigilancia Epidemiológica (2010) Sistema único de información. Boletín Epidemiología 34:1-27.

Stepp J. R. (2004) The role of weeds as sources of pharmaceuticals. Journal of Ethnopharmacology 92:163-166.

Stepp J. R. and D. E. Moerman (2001) The importance of weeds in ethnopharmacology. Journal of Ethnopharmacology 92:163-166.

Teklehaymanot T. (2009) Ethnobotanical study of knowledge and medicinal plants use by the people in Dek Island in Ethiopia. Journal of Ethnopharmacology 124:69-78.

Valdés J. (1974) Los jardines botánicos. Revista de la Universidad de México 29:11-16.

Voeks R. A. (1996) Tropical forest healers and habitat preference. Economic Botany 50:381-400.

Vovides A., E. Linares y R. Bye (2010) Jardines Botánicos de México: Historia y Perspectiva. Ed. Secretaría de Educación de Veracruz. Xalapa, Veracruz, México. 232 p.

Weckerle C. S., R. Ineichen, F. K. Huber and Y. Yang (2009) Mao's heritage: Medicinal plant knowledge among the Bai in Shaxi, China, common widespread practice. Journal of Ethnopharmacology 123:213-228.

Weniger B., M. Haag-Berrurier and R. Anton (1982) Plants of Haiti used as antifertility agents. Journal of Ethnopharmacology 6:67-84.

Zolla C., S. Del-Bosque, A. Tascón y V. Mellado (1988) Medicina tradicional y enfermedad. Ed. Centro Interamericano de Estudios de Seguridad Social, Instituto Mexicano del Seguro Social. México. 146 p. 